\title{
Influence of oral mucosal lesions and oral symptoms on oral health related quality of life in dermatological patients: a cross sectional study in Sudan
}

Nada M Suliman ${ }^{1 *}$, Anne C Johannessen ${ }^{1,2}$, Raouf W Ali ${ }^{3}$, Hussein Salman ${ }^{4}$ and Anne N Åstrøm ${ }^{5}$

\begin{abstract}
Background: There are only few studies considering the impact of oral mucosal lesions (OML) on the oral quality of life of patients with different dermatological conditions. This study aimed to assess the relationship between oral health-related quality of life (OHRQoL) and OML and reported oral symptoms, perceived general and oral health condition and caries experience in adult skin diseased patients attending an outpatient dermatologic clinic in Sudan.
\end{abstract}

Methods: A cross-sectional survey was carried out with 544 diagnosed skin diseased patients (mean age 37.1 years, $50 \%$ females), during the period October 2008 to January 2009. The patients were orally examined and OML and caries experience was recorded. The patients were interviewed using the Sudanese Arabic version of the OIDP. OHRQoL was evaluated by socio-demographic and clinical correlates according to number of types of OML diagnosed (no OML, one type of OML, > one type of OML) and number and types of oral symptoms.

Results: An oral impact (OIDP >0) was reported by 190 patients (35.6\%) (mean OIDP total score 11.6, sd =6.7). The prevalence of any oral impact was $30.5 \%, 36.7 \%$ and $44.1 \%$, in patients with no OML, one type of OML and more than one type of OML, respectively. Number of types of OML and number and types of oral symptoms were consistently associated with the OIDP scores. Patients who reported bad oral health, patients with $\geq 1$ dental attendance, patients with $>1$ type of $\mathrm{OML}$, and patients with $\geq 1$ type of oral symptoms were more likely than their counterparts in the opposite groups to report any OIDP. The odds ratios (OR) were respectively; 2.9 (95\% Cl 1.9-4.5), 2.3 (95\% Cl 1.5-3.5), 1.8 (95\% Cl 1.1-3.2) and 6.7 (95\% Cl 2.6-17.5). Vesiculobullous and ulcerative lesions of OML disease groups associated statistically significantly with OIDP.

Conclusion: OIDP was more frequently affected among skin diseased patients with than without OML. The frequency of the impacts differed according to the number of type of OML, oral symptoms, and OML disease groups. Dentists and dermatologists should pay special attention to skin diseased patients because they are likely to experience oral impacts on daily performances.

Keywords: dermatology, oral mucosal lesions, oral impact on daily performance, quality of life

\footnotetext{
* Correspondence: Nada.Suliman@gades.uib.no

'Section for pathology, The Gade Institute, University of Bergen, Bergen, Norway

Full list of author information is available at the end of the article
} 


\section{Background}

Oral mucosal lesions (OML) may be the initial feature or the only clinical sign of mucocutaneous diseases, a group of mainly chronic diseases, commonly observed in a dermatologic practice [1-4]. In a previous study considering Sudanese adults with mucocutaneous diseases attending an outpatient dermatology clinic in Khartoum, the prevalence of patients with OML was high, amounting to $57.9 \%$ [5]. Patients with OML experience a wide range of chronic and recurrent conditions that may have detrimental effect on functioning, social life and psychological well-being.

Evidently, mucocutaneous diseases have impacts on the quality of life of patients comparable to that of other medical conditions [6,7]. Patient reported outcomes in terms of oral health related quality of life (OHRQoL) measures have seldom been assessed in relation to mucocutaneous conditions [8-15]. Whereas the relationship of dental and periodontal status with OHRQoL measures has been examined across various socio-cultural contexts, few studies have considered the impact on OHRQoL of patients with disorders that are of relevance to oral medicine and dermatological practice $[11,13,16,17]$. This is so, although patient reported outcomes of OHRQoL may provide valuable information, for example by identifying treatment needs, selecting therapies, evaluating treatment outcomes and monitoring patient progress [18].

Several generic and disease specific OHRQoL measures have been developed to provide better understanding of the consequences of oral diseases upon quality of life and to complement traditional clinical measures $[6,19]$. Whereas specific OHRQoL measures assess impacts that are attributable to specific oral diseases, the generic ones take into account numerous oral conditions, some occurring simultaneously, thus providing information on the wider implications of oral status [20]. One promising generic OHRQoL measure is the Oral Impacts on Daily Performance (OIDP) scale $[21,22]$. The OIDP was developed to measure oral impacts that seriously affect a person's daily life. It is based on the conceptual framework of the World Health Organisation's International Classification of Impairments, Disabilities and Handicaps (ICIDH) [23], which has been amended for dentistry by Locker [24]. The OIDP concentrates only on the measurement of "ultimate" oral impacts, thus covering the fields of disability and handicap [22] . This inventory assesses the impact of oral conditions on basic activities and behaviours that cover the physical, psychological, and social dimensions of daily living. Considering respondent burden, the OIDP is suitable for use in population surveys and clinical practices, not only in terms of being easier when measuring behaviours rather than feeling states, but also in being short. It is originally calculated by multiplying frequency and severity scores of daily performances, providing an overall score for each OIDP item. However, applications of the weighted
OIDP scores revealed no significant improvement over the use of OIDP frequency or severity scores [22]. Thus, it has been proposed to use either the frequency or the severity OIDP scores for simplicity and efficiency. Since its development, the OIDP has shown to be reliable and valid in general population based studies [25-28], as well as in studies of patients with specific oral disorders, such as traumatic injuries, periodontal disease and malocclusion $[16,17,29]$. Although an Arabic version of the 8 item OIDP inventory has been applied previously with Sudanese children [30] and dental attendees from a Sudanese adult population [31], this study necessitated reestablishment of its psychometrical properties. The generic OIDP inventory has yet to be applied in the context of patients with mucocutaneous diseases.

This study aimed to assess the relationship between oral health related quality of life (OHRQoL) and OML and reported oral symptoms, perceived general and oral health condition and caries experience in Sudanese adult skin diseased patients attending an outpatient dermatologic clinic in Sudan.

\section{Methods}

\section{Sampling procedure}

The present study is a part of a cross sectional hospital based study that was carried out from October 2008 to January 2009 [5]. The study was focusing on patients aged 18 years and above with mucocutaneous diseases, attending an outpatient dermatologic clinic at Khartoum Teaching Hospital $(\mathrm{KTH}) . \mathrm{KTH}$ is the largest national hospital in Sudan, located in Khartoum, the capital city. It is an open public and referral hospital receiving patients from all states of the country. A minimum sample size of 500 patients was calculated to estimate differences in OHRQoL between patients with and without oral mucosal lesions assuming the proportions of oral impacts to be 0.60 and 0.40 among patients with and without OML lesions, significance level (two sided test) of $5 \%$ and statistical power of $80 \%$. All patients $(n=4235)$ attending the outpatient facility during the survey period were invited to participate in the study. A total of 1540 subjects (36.4 \%) initially accepted to participate. Fear of taking biopsy for asymptomatic lesions and time consuming examinations (oral examination, interview, and biopsy when needed) were the main reasons for not volunteering to participate. Of those who initially accepted to participate, 544 (544/ 1540, $35.3 \%)$ patients were included in the study. Unexplained disappearance of patients and limited financial resources were the main reasons for withdrawal from the study. Thus, the final participation rate was 544/4235, $12.8 \%$. Confidentiality of the patients was maintained, participants were informed about their oral conditions, and health education was provided. Those who needed dental services were referred to the University of Science and 
Technology (UST), Faculty of Dentistry, for further investigation and management. Written informed consent or finger print (illiterates) for participation and publication of the study was obtained from patients or their parents/guardians. The research conformed to the Helsinki Declaration, and ethical clearance and approval letters were obtained by the participating institutions' committees in Sudan (UST and KTH, Department of Dermatology). In Norway, the ethical approval was obtained from the Regional Committee for Medical Research Ethics of Western Norway.

\section{Survey instrument}

A pilot study revealed a considerable number of illiterate patients, and thus a structured questionnaire was interviewer administered by two trained dentists. The interview schedule contained questions regarding socio-demographics, health and oral health related characteristics and lifestyles. The interview schedule was constructed in English and then translated and used in Arabic. Forward and backward translations were performed by two independent Sudanese professional translators in Arabic and English language. Sensitivity to culture and selection of appropriate words were considered by use of simple common Arabic words.

OHRQoL was assessed using the eight items OIDP frequency inventory [21,22]; 'During the past 6 months, how often have problems with your mouth and teeth caused you any difficulty with: eating and chewing food; speaking and pronouncing clearly; cleaning teeth; sleeping and relaxing; smiling and showing teeth without embarrassment; maintaining usual emotional state; carrying out major work and social role, and enjoying contact with people?'. Each item was assessed using a 5-point scale: (1) Never affected; (2) Less than once a month; (3) Once or twice a month; (4) Once or twice a week; (5) Every, or nearly every day. Initially, an additive sum score (OIDP ADD) was constructed from the 8 items as originally scored (1-5, range $8-40)$. Secondly, each OIDP frequency item was dichotomised, yielding the categories: (0) never affected (including the original category 1), (1) affected (including the original categories 2, 3, 4, and 5). Simple count scores (SC) were created for the OIDP by adding the eight dichotomised variables. For the purpose of cross-tabulation and logistic regression analysis, the OIDP SC scores (0-8) were dichotomised as $0=$ no daily performance affected and $1=$ at least one daily performance affected. The distribution of the OIDP SC scores supported this cut-off point.

Socio-demographic characteristics were assessed in term of gender, age, education, tribes, marital status, and place of residence. Gender was assessed as: (1) female; (2) male. Age was recorded by asking, 'how old are you?' and the answers were dichotomized into 2 equally sized groups; (0) 18-32 years and (1) 33 + years. Participants were classified according to their educational level using five categories: (1) illiterate; (2) primary school; (3) secondary school;
(4) university; (5) higher studies. Two dummy variables were constructed yielding the categories $0=$ lower education (including the original categories 1 and 2) and 1 = higher education (including the original categories 3, 4, and 5). Medical condition was assessed as a sum score of the following: heart diseases, hypertension, asthma, diabetes, liver diseases, hepatitis /jaundice, anaemia, bleeding disorders, kidney diseases, rheumatoid arthritis, allergy, cancer, epilepsy, stomach ulcer, intestinal disorders, psychiatric/mental disorders, respiratory disorders, and pregnancy. The sum scores were dichotomized into $0=$ none and $1=\geq$ one. Perceived health status was recorded from (1) very bad to (4) very good. Two dummy variables were created in terms of $0=$ good and $1=$ bad. Perceived oral health status was measured using a 5-point rating by asking; 'How do you consider the present condition of your mouth and teeth?' with response categories: (1) very bad; (2) bad; (3) neither good nor bad; (4) good; (5) very good. This variable was dichotomized in terms of $0=\operatorname{good}(i n-$ cluding the original categories 4 and 5) and (1) = bad (including the original categories 1, 2 and 3). Reported oral symptoms were assessed by the question 'During the previous 6 months have you experienced: dental pain/toothache, abscessed tooth, dry mouth, bleeding gums, infected sore gums, tooth decay, or broken tooth. Each symptom was assessed as present (1) and absent (0). Frequency of dental attendance was assessed by asking 'How many times have you attended a dentist during the previous 2 years?' with response categories: (1) once; (2) twice, (3) more than twice; (4) never. A dummy bivariable was constructed yielding the response categories $1=$ attended dental clinic (including the original categories 1,2 and 3) and $0=$ never attended dental clinic.

\section{Clinical examination}

Systematic comprehensive extra-oral and intra-oral clinical examinations based on visual inspection and palpation, following the World Health Organization (WHO) criteria for field surveys [32], were carried out by a dentist (NMS) who received a standard training in diagnosis of OML before the data collection (The Gade Institute, Section for Pathology, and Department of Clinical Dentistry, Section for Oral Surgery and Oral Medicine, University of Bergen, Norway). Caries experience was assessed under field conditions and scored according to the criteria described by the WHO [33]. A tooth was recorded as decayed when a cavity was apparent on visual inspection. Missing tooth was recorded if there was a history of extraction because of pain and/ or a cavity prior to extraction. DMFT, was computed as the sum of decayed, missing and filled teeth and dichotomized into caries free $\mathrm{DMFT}=0$ and having any caries experience DMFT $>0$. The oral clinical examination and information with respect to OML and oral habits have been detailed elsewhere [5]. 
Table 1 Socio-demographics, behavioural- and clinical characteristics of patients with mucocutaneous diseases according to number of types of OML

\begin{tabular}{|c|c|c|c|c|}
\hline & No OML\% (n) & One type of OML\% (n) & $>$ One type of OML\% (n) & Total\% (n) \\
\hline \multicolumn{5}{|l|}{ Gender } \\
\hline Female & $56.3(129)$ & $49.5(100)$ & $38.1(43)$ & $50.0(272)$ \\
\hline Male & $43.7(100)$ & $50.5(102)$ & $61.9(70)$ & $50.0(272)$ \\
\hline \multicolumn{5}{|l|}{ Age } \\
\hline Younger (18-32 years) & $57.9(132)$ & $53.3(105)$ & $37.8(42)$ & $52.2(279)$ \\
\hline Older (33-85 years) & $42.2(96)$ & $46.2(90)$ & $62.2(69)$ & $47.8(255)$ \\
\hline \multicolumn{5}{|l|}{ Education } \\
\hline Low & $46.3(105)$ & $49.5(97)$ & $58.0(65)$ & $49.9(267)$ \\
\hline High & $53.7(122)$ & $50.5(99)$ & $42.0(47)$ & $50.1(268)$ \\
\hline \multicolumn{5}{|l|}{ Systemic condition } \\
\hline None & $62.4(143)$ & $50.0(101)$ & $46.9(53)$ & $54.6(297)$ \\
\hline$\geq$ one & $37.6(86)$ & $50.0(101)$ & $53.1(60)$ & $45.4(247)$ \\
\hline \multicolumn{5}{|l|}{ Perceived general health status } \\
\hline Bad & $31.1(71)$ & $27.1(54)$ & $44.1(49)$ & $32.3(174)$ \\
\hline Good & $68.9(157)$ & $72.9(145)$ & $55.9(62)$ & $67.7(364)$ \\
\hline \multicolumn{5}{|l|}{ Perceived oral health status } \\
\hline Bad & $36.0(82)$ & $39.5(79)$ & $36.6(41)$ & $37.4(202)$ \\
\hline Good & $64.0(146)$ & $60.5(121)$ & $63.4(71)$ & $62.6(338)$ \\
\hline \multicolumn{5}{|l|}{ Dental attendance } \\
\hline Never attended dental clinic & $67.3(152)$ & $60.0(120)$ & $52.2(59)$ & $61.6(331)$ \\
\hline Attended dental clinic & $32.7(74)$ & $39.4(78)$ & $47.8(54)$ & $38.4(206)$ \\
\hline \multicolumn{5}{|c|}{ Number of reported oral symptoms } \\
\hline None & $18.2(38)$ & $15.0(26)$ & $11.2(11)$ & $15.6(75)$ \\
\hline$\geq$ one & $81.8(171)$ & $85.0(147)$ & $88.8(87)$ & $84.4(405)$ \\
\hline \multicolumn{5}{|l|}{ Specific reported oral symptoms } \\
\hline Dental pain (yes) & $44.6(100)$ & $45.2(89)$ & $48.6(54)$ & $45.7(243)$ \\
\hline Tooth decay (yes) & $58.7(132)$ & $50.8(101)$ & $65.5(72)$ & $57.1(305)$ \\
\hline Abscess (yes) & $9.0(20)$ & $9.6(19)$ & $10.7(12)$ & $9.6(51)$ \\
\hline Broken tooth (yes) & $14.4(32)$ & $19.1(36)$ & $11.4(12)$ & $15.5(80)$ \\
\hline Dry mouth (yes) & $21.3(48)$ & $22.1(43)$ & $22.0(24)$ & $21.7(115)$ \\
\hline Bleeding gum (yes) & $36.4(83)$ & $37.9(75)$ & $44.1(49)$ & $38.5(207)$ \\
\hline Infected sore gum (yes) & $12.4(28)$ & $16.7(32)$ & $23.4(26)$ & $16.3(86)$ \\
\hline OML pain (yes) & $1.3(3)$ & $19.3(39)$ & 11.5 (13) & $10.1(55)$ \\
\hline \multicolumn{5}{|l|}{ DMFT } \\
\hline $\mathrm{DMFT}=0$ & $8.7(20)$ & $12.9(26)$ & $8.0(9)$ & $10.1(55)$ \\
\hline $\mathrm{DMFT}>0$ & $91.3(209)$ & 87.1 (176) & $92.0(104)$ & $89.9(489)$ \\
\hline
\end{tabular}

The total number in the different categories did not add to 544 owing to missing values.

\section{Diagnostic criteria for oral mucosal lesions}

An OML was defined as any abnormal change or any swelling on the oral mucosal surface. A single lesion with confirmed diagnosis was referred to as a 'type of OML'. Diagnostic criteria for OML were based on Axéll criteria and those defined in earlier studies and reviews [32,34,35].

\section{Statistical analysis}

Data were analysed using PASW Statistics version 18.0 (SPSS Inc., Chicago). Non-parametric statistics were used because the OIDP-total scores were not normally distributed. Bivariate relationships were assessed using crosstabulation, chi-square statistics and Mann Whitney - U 
Table 2 Percentage distribution and mean scores (SD) for the eight OIDP frequency items and the OIDP ADD score in skin diseased patients by number of types of $\mathrm{OML}$

\begin{tabular}{|c|c|c|c|c|c|c|c|c|}
\hline \multirow[t]{2}{*}{ OIDP items } & \multicolumn{2}{|c|}{ No OML N= 229} & \multicolumn{2}{|c|}{ One type of OML N=202 } & \multicolumn{2}{|c|}{$>$ One type of OML N=113 } & \multicolumn{2}{|c|}{ Total population $\mathrm{N}=544$} \\
\hline & Affected\% (n) & Mean (SD) & Affected\% (n) & Mean (SD) & Affected\% (n) & Mean (SD) & Affected\% (n) & Mean (SD) \\
\hline Eating & $26.4(60)$ & $1.7(1.4)$ & $33.0(66)$ & $2.0(1.6)$ & $39.8(45)$ & $2.2(1.6)$ & $31.7(171)$ & $1.9(1.5)$ \\
\hline Emotional state & $16.2(37)$ & $1.5(1.2)$ & $25.9(51)$ & $1.8(1.4)$ & $30.4(34)$ & $1.9(1.5)$ & $22.7(122)$ & $1.7(1.3)$ \\
\hline Cleaning & $17.1(39)$ & $1.5(1.2)$ & $23.4(46)$ & $1.7(1.4)$ & $26.8(30)$ & $1.8(1.5)$ & $21.4(115)$ & $1.6(1.3)$ \\
\hline Sleeping & $12.7(29)$ & $1.3(1.0)$ & $18.3(36)$ & $1.5(1.2)$ & $16.2(18)$ & $1.5(1.2)$ & $15.5(83)$ & $1.4(1.1)$ \\
\hline Speaking & $4.80(11)$ & $1.1(0.6)$ & $8.0(16)$ & $1.2(0.8)$ & $15.9(18)$ & $1.4(1.1)$ & $8.3(45)$ & $1.2(0.8)$ \\
\hline Contact people & $4.40(10)$ & $1.1(0.6)$ & $9.5(19)$ & $1.3(1.0)$ & $10.6(12)$ & $1.3(1.0)$ & $7.6(41)$ & $1.2(0.9)$ \\
\hline Major work & $4.40(10)$ & $1.1(0.6)$ & $7.7(15)$ & $1.2(0.9)$ & $9.8(11)$ & $1.3(1.0)$ & $6.7(36)$ & $1.2(0.8)$ \\
\hline Smiling & $1.8(4)$ & $1.0(0.4)$ & $8.5(17)$ & $1.2(0.8)$ & $11.5(13)$ & $1.4(1.2)$ & $6.3(34)$ & $1.2(0.8)$ \\
\hline $\mathrm{OIDP}>0$ & $30.5(69)$ & $0.8(1.5)$ & 36.7 (72) & $1.3(2.1)$ & 44.1 (49) & $1.5(2.3)$ & $35.6(190)$ & $1.1(1.9)$ \\
\hline OIDP ADD & & $10.4(4.8)$ & & $12.1(7.2)$ & & $13.1(8.4)$ & & $11.6(6.7)$ \\
\hline
\end{tabular}

Means and \% varied according to the total number of respondents in each OIDP item due to lack of information in 2-11 patients across the OIDP items.

test. Internal consistency reliability was assessed using Cronbach's alpha. To adjust for potential confounding factors, multiple variable logistic regression analyses were performed and OR and Nagelkerkes $R^{2}$ were calculated. The relationship between OIDP and number of different types of OML was assessed in unadjusted and fully adjusted models. The relationship between OIDP and each type of reported symptoms and OML disease groups was assessed in unadjusted, fully adjusted and mutually adjusted models.

\section{Result}

\section{Sample profile}

A total of 544 patients with mucocutaneous diseases participated in the present study. The mean age was 37.1 years, $\mathrm{sd}=15.9$ years (range 18-85), $50 \%$ were females, $77 \%$ were permanent residents of Khartoum during the previous 5 years, $47.8 \%$ belonged to the older age group (33-85 years) and $50.1 \%$ reported higher education. A total of $57.9 \%$ of the patients were diagnosed with at least one clinically recognized type of OML. Full details of the prevalence of OML (types and group diseases) of the participants studied are described elsewhere [5]. A particular type of OML was recorded only once although it could be manifested at several locations in the same patient. The age of patients affected by OML ranged from 18 to 81 years, with an average of 38.6 years $(\mathrm{sd}=16.5)$. A total of $6 \mathrm{OML}$ group diseases, each including at least 20 patients, were recognized for the present study. Tongue lesions were the most frequently diagnosed OML group diseases $(23.3 \%)$ followed in descending order by white lesions (19.1\%), red and blue lesions (11\%), vesiculobullous diseases (6\%), oral ulcerative lesions (4.5\%) and pigmented lesions (3.9\%). Table 1 depicts the distribution of patients' socio-demographic, behavioural, oral symptoms and clinical features by number of types of OML. As shown, a total of $89.9 \%$ had caries experience, $84.4 \%$ reported more than one oral symptom and $45.5 \%$ reported more than one systemic health condition. The most and least frequently reported conditions were tooth decay $(57.1 \%)$ and abscess $(9.6 \%)$, respectively.

\section{Psychometric properties of the OIDP}

In the present study, small number of missing responses (211) adds support to face validity of the OIDP frequency inventory. As depicted in Table 2, One hundred and ninety patients $(35.6 \%)$ perceived at least one oral impact (OIDP $>0)$. The mean OIDP ADD was $11.6(\mathrm{sd}=6.7)$ The prevalence of any oral impact was $30.5 \%, 36.7 \%$ and $44.1 \%$ in patients with respectively, no OML, one type of OML and more than one type of OML. A problem with eating was the most frequently reported impact. Problems with work, contact people, and smiling were the least frequently reported impacts across the three OML groups as well as in the total study group. As shown in Table 3, Cronbach's alpha for the OIDP in the study group was 0.89 with corrected item-total correlation ranging from 0.57 (smiling) to 0.70 (emotional state). The standardized items alpha in the separate groups was 0.81 (no OML), 0.89 (one type of OML) and 0.92 (> one type of OML). The corrected item-total correlation across the three groups was above the minimum level of 0.2 required for including an item into a scale [36].

The association between the frequency of oral impacts (OIDP total $>0$ ) and factors known to be associated with oral health; socio-demographic-, clinical and behavioural variables were assessed using cross tabulation and multiple variable logistic regression analyses. As depicted in Table 4, the frequency of subjects having at least one impact (OIDP $>0$ ) increased significantly with increasing number of types of OML both in unadjusted and adjusted analysis with subjects having more than one type of OML being about twice as likely as their counterparts without OML to report oral 
Table 3 Corrected item total correlation and Cronbach's alpha of OIDP by number of types of OML

\begin{tabular}{lll}
\hline No OML (N=226) Cronbach's Alpha (Standardized Items) $=\mathbf{0 . 8 1 1}$ \\
\hline OIDP items & $\begin{array}{l}\text { Corrected Item-Total } \\
\text { Correlation }\end{array}$ & $\begin{array}{l}\text { Cronbach's Alpha } \\
\text { if Item Deleted }\end{array}$ \\
\hline Eating & .675 & .757 \\
Speaking & .447 & .792 \\
Cleaning & .592 & .767 \\
Smiling & .269 & .808 \\
Sleeping & .634 & .759 \\
Emotional state & .655 & .755 \\
Carrying out major work & .564 & .779 \\
Contact & .391 & .796 \\
One type of OML (N=196) & \\
Cronbach's Alpha (Standardized Items)=0.894 & \\
Eating & .721 & .867 \\
Speaking & .586 & .878 \\
Cleaning & .713 & .865 \\
Smiling & .614 & .876 \\
Sleeping & .715 & .865 \\
Emotional state & .680 & .870 \\
Carrying out major work & .646 & .874 \\
Contact & .683 & .870 \\
\end{tabular}

> One type of OML $(\mathrm{N}=111)$

Cronbach's Alpha (Standardized Items) $=0.921$

\begin{tabular}{lll} 
Eating & .700 & .909 \\
Speaking & .711 & .906 \\
Cleaning & .751 & .902 \\
Smiling & .657 & .910 \\
Sleeping & .751 & .902 \\
Emotional state & .779 & .900 \\
Carrying out major work & .766 & .903 \\
Contact & .747 & .904 \\
Total study population (N $=533)$ & \\
Cronbach's Alpha (Standardized Items) $=0.890$ & \\
Eating & .694 & .863 \\
Speaking & .606 & .869 \\
Cleaning & .692 & .859 \\
Smiling & .572 & .872 \\
Sleeping & .698 & .858 \\
Emotional state & .703 & .858 \\
Carrying out major work & .671 & .864 \\
Contact & .642 & .866 \\
\hline
\end{tabular}

impacts (OR 1.8 (95 \% CI 1.1-3.2). Perceived oral health status remained statistically significantly associated with OIDP after having included all variables in the model. Multiple logistic regression analysis revealed that socio-demographic, behavioural variables and medical conditions entered in the first step explained $20 \%$ of the variance (Nagelkerke $\left.R^{2}=0.20\right)$. Entering number of types of OML in step 2 raised the explainable variance by $1 \%$ (Nagelkerke $R^{2}=0.21$ ).

As shown in Table 5, six out of eight specific symptoms were associated with impaired OHRQoL in adjusted logistic regression analyses. When all reported symptoms were accounted for, only OML pain (OR 10.3, 95 \% CI 4.2-25.4), infected sore gums (OR 4.1, $95 \%$ CI 1.9-8.6), dental pain (OR 3.1, 95 \% CI 1.8-5.3) and tooth decay (OR 1.8, 95 \% CI 1.0-3.1) remained statistically significantly associated with OIDP. Pain associated with mucosal lesion had the strongest impact OR 10.2 (95\% CI 4.2-25.0), and dry mouth the weakest impact OR 0.9 (95\% CI 0.4-1.7) on OIDP. As depicted in Table 6, the OML disease groups of vesiculobullous and ulcerative lesions discriminated statistically significantly between subjects with and without OIDP in adjusted as well as in mutually adjusted logistic regression analyses. A total of $72.4 \%$ versus $33.5 \%(\mathrm{p}<0.001)$ of the participants with and without vesiculobullous lesions and $77.3 \%$ versus $33.9 \%$ of participants with and without oral ulcerative lesions $(\mathrm{p}<0.001)$ had oral impacts on their daily performances. When adjusting for socio-demographics, subjects with vesiculobullous lesions were 7.4 times OR 7.4 (95\% CI 2.9-18.8) and subjects with oral ulcerative lesions were 5.7 times OR 5.7 (95\% CI 1.9-16.9) more likely than their counterparts without those OML disease groups to report oral impacts. The corresponding mutually adjusted ORs were 8.2 (95 \% CI 3.2-20.9) and 6.7 (95 \% CI 2.2-20.0).

\section{Discussion}

This is the first study considering OHRQoL in patients with various mucocutaneous diseases, using an Arabic version of the OIDP frequency inventory. Arabic versions of OHRQoL instruments such as the Oral Health Impact Profile (OHIP-14), the Geriatric Oral Health Assessment Index (GOHAI) and the OHQoL-UK inventory have been reported to be reliable and valid for use in adult populations from Saudi Arabia, Egypt and Syria [37-39]. The results of this study indicate that, when used with patients having mucocutaneous diseases, the Arabic OIDP version is valid and reliable demonstrating psychometric properties similar to the original English version [27] as well as the Thai [26], Greek [28] and Norwegian versions of the OIDP [40]. Moreover, the OIDP has shown to be usable across various subgroups of the Sudanese population [30,31], first applied as a self-administered questionnaire in dental attendees from the general population, secondly in personal interviews with schoolchildren and more recently in personal interviews with patients in a dermatologic clinic. Thus, internal consistency reliability in terms of Cronbach's alphas of 0.89 was satisfactory and well above the recommended level of 0.70 [36]. Moreover, the corrected item-total correlation coefficients were above the 
Table 4 Unadjusted and adjusted associations of OIDP with socio-demographics, behaviours and number of types of OML in skin diseased patients $(n=544)$. Percentage $(n)$, odds ratio (OR) and $95 \%$ Confidence Interval $(\mathrm{Cl})$

\begin{tabular}{|c|c|c|c|}
\hline Variables & OIDP $>0 N=190 \%(n)$ & Unadjusted OR (95\% Cl) & Adjusted OR (95\% Cl) \\
\hline \multicolumn{4}{|l|}{ Gender } \\
\hline Male & $30.7(81)$ & 1 & 1 \\
\hline Female & $40.5(109)^{*}$ & $1.5(1.0-2.2)$ & $1.4(0.9-2.1)$ \\
\hline \multicolumn{4}{|l|}{ Age } \\
\hline $18-32 \mathrm{yr}$ & $34.7(95)$ & 1 & 1 \\
\hline $33-85 \mathrm{yr}$ & $36.4(91)$ & $1.0(0.7-1.5)$ & $0.9(0.6-1.5)$ \\
\hline \multicolumn{4}{|l|}{ Education } \\
\hline Lower & $31.2(82)$ & 1 & 1 \\
\hline Higher & $39.3(103)^{*}$ & $1.4(0.9-2.0)$ & $1.4(0.9-2.3)$ \\
\hline \multicolumn{4}{|l|}{ Perceived oral health status } \\
\hline Good & $24.5(82)$ & 1 & 1 \\
\hline Bad & $54.8(108)^{* *}$ & $3.7(2.5-5.4)$ & $2.9(1.9-4.5)^{* *}$ \\
\hline \multicolumn{4}{|l|}{ Perceived health status } \\
\hline Good & $30.5(110)$ & 1 & 1 \\
\hline Bad & $46.7(79) * *$ & $2.0(1.3-2.9)$ & $1.5(0.99-2.4)$ \\
\hline \multicolumn{4}{|l|}{ Dental attendance } \\
\hline Never attended dental clinic & $27.4(90)$ & 1 & 1 \\
\hline Attended dental clinic & $48.8(98)^{* *}$ & $2.5(1.7-3.6)$ & $2.3(1.5-3.5)^{* *}$ \\
\hline \multicolumn{4}{|l|}{ Medical conditions } \\
\hline None & $30.1(87)$ & 1 & 1 \\
\hline At least one & $42.2(103)^{*}$ & $1.6(1.1-2.4)$ & $1.3(0.8-2.1)$ \\
\hline \multicolumn{4}{|l|}{ Number of types of OML \# } \\
\hline None & 30.5 (69) & & 1 \\
\hline One & 36.7 (72) & & $1.2(0.7-1.9)$ \\
\hline >one & $44.1(49)^{*}$ & & $\begin{array}{l}1.8 \\
(1.1-3.2)^{*}\end{array}$ \\
\hline
\end{tabular}

$\left.{ }^{*} \mathrm{p}<0.05,{ }^{* *} \mathrm{p}<0.001, \uparrow \mathrm{p}=0.05\right)$

\# Number of types of OML; no OML $(p=0.06)$, one type OML $(p=0.3)$, >one type OML $(p=0.02)$

The sum of the categories listed may not equal the total number due to lack of information.

minimum level of 0.20 for inclusion of an item into a scale across patients with and without OML [36]. Although no approach guarantees cross-cultural equivalence, the Arabic version of OIDP seemed to preserve the overall concepts of the English version and did not differ in terms of sequence of questions, the Likert scale and the recall memory period (6 months) used. Notably, the respondents had few difficulties in completing the 8 item OIDP interview. This highlights the feasibility of employing the Arabic version of the OIDP frequency inventory in oral medicine and dermatologic clinical settings in Sudan. Recognizing the frequency and severity of the OIDP scores to have similar predictive power, using the OIDP frequency score in this study, should be the better single choice because of its better reproducibility [22]. However, the degree of impact could not be accounted for by this model.

According to the present results, the frequency of oral impacts varied systematically and in the expected direction with self-reported oral health status, clinical dentition status and number of reported oral symptoms across patients having none, at least one and more than one type of OML. Moreover, patients having more than one type of OML were more likely to report oral impacts than their counterparts without OML and with only one type of OML, suggesting a cause - effect relationship. Notably, cross-sectional studies cannot provide definite information about cause - and- effect relationships since both predictor and outcome variables have been measured at the same point in time. Longitudinal studies are needed to improve the interpretation of factors influencing OIDP in adult patients with OML. The moderate fit of the overall multivariable model indicates that other essential variables were not included in the model. Types of OML have fluctuated from asymptomatic lesions (snuff dipper lesions) to the most chronic and painful one (oral pemphigus vulgaris) [5]. In the 
Table 5 Unadjusted, adjusted and mutually adjusted associations of OIDP with reported oral symptoms in skin diseased patients $(n=544)$. Percentages $(n)$, odds ratios (OR) and $95 \%$ confidence interval $(C I)$

\begin{tabular}{|c|c|c|c|c|}
\hline $\begin{array}{l}\text { Reported symptoms } \\
\text { previous } 6 \text { months }\end{array}$ & OIDP $>0 \%(n)$ & $\begin{array}{l}\text { Unadjusted OR } \\
(95 \% \mathrm{Cl})\end{array}$ & $\begin{array}{l}\text { Adjusted a }{ }^{\circ} \\
(95 \% \mathrm{Cl})\end{array}$ & $\begin{array}{l}\text { Mutually adjusted }{ }^{\mathrm{b}} \mathrm{OR} \\
(95 \% \mathrm{Cl})\end{array}$ \\
\hline \multicolumn{5}{|l|}{ Dental pain } \\
\hline No & $20.0(57)$ & 1 & 1 & 1 \\
\hline Yes & $55.2(132)^{* *}$ & $4.9(3.3-7.2)$ & $4.3(2.8-6.6)^{* *}$ & $3.1(1.8-5.3)^{* *}$ \\
\hline \multicolumn{5}{|l|}{ Tooth decay } \\
\hline No & $21.8(49)$ & 1 & 1 & 1 \\
\hline Yes & $46.4(140)^{* *}$ & $3.1(2.1-4.5)$ & $2.8(1.8-4.3)^{* *}$ & $1.8(1.0-3.1)^{*}$ \\
\hline \multicolumn{5}{|l|}{ Abscess } \\
\hline No & $31.7(150)$ & 1 & 1 & 1 \\
\hline Yes & $74.0(37)^{* *}$ & $6.1(3.1-11.8)$ & $4.7(2.3-9.5)^{* *}$ & $2.3(1.0-5.3)^{*}$ \\
\hline \multicolumn{5}{|l|}{ Broken tooth } \\
\hline No & $36.2(155)$ & 1 & 1 & \\
\hline Yes & $35.4(28)$ & $0.9(0.5-1.5)$ & $0.8(0.4-1.4)$ & - \\
\hline \multicolumn{5}{|l|}{ Dry mouth } \\
\hline No & $32.7(133)$ & 1 & 1 & 1 \\
\hline Yes & $46.5(53)^{*}$ & $1.7(1.1-2.7)$ & $1.5(0.9-2.4)$ & $0.9(0.4-1.7)$ \\
\hline \multicolumn{5}{|l|}{ Bleeding gum } \\
\hline No & $27.0(88)$ & 1 & 1 & 1 \\
\hline Yes & $49.3(100)^{* *}$ & $2.6(1.8-3.7)$ & $2.5(1.6-3.7)^{* *}$ & $1.3(0.7-2.2)$ \\
\hline \multicolumn{5}{|l|}{ Infected sore gum } \\
\hline No & $27.8(122)$ & 1 & 1 & 1 \\
\hline Yes & $78.0(64)^{* *}$ & $9.2(5.2-16.2)$ & $7.4(4.0-13.4)^{* *}$ & $4.1(1.9-8.6)^{* *}$ \\
\hline \multicolumn{5}{|l|}{ OML pain } \\
\hline No & $31.3(151)$ & 1 & 1 & 1 \\
\hline Yes & $76.5(39)^{* *}$ & $7.1(3.6-13.9)$ & $11.2(5.2-23.9)^{* *}$ & $10.3(4.2-25.4)^{* *}$ \\
\hline \multicolumn{5}{|l|}{ Number of symptoms } \\
\hline None & $9.6(7)$ & 1 & 1 & \\
\hline At least one & $41.0(164)^{* *}$ & $6.5(2.9-14.6)$ & $6.7(2.6-17.5)^{* *}$ & \\
\hline
\end{tabular}

${ }^{*} \mathrm{p}<0.05,{ }^{* *} \mathrm{p}<0.001$

a) Adjusted for sex, age, education, perceived health status, dental attendance and medical condition.

b) Adjusted for sex, age, education, perceived health status, dental attendance, medical condition and other symptoms.

The sum of the categories listed may not equal the total number due to lack of information.

future, stratified analysis of types of OML should be considered as chronic OML has proven to decrease quality of life [41].

Both type and number of reported oral symptoms discriminated between patients with and without oral impacts (OIDP $>0)$. Dental attendance was one of the strongest predictors of oral impact in this study.The association between dental attendance and improved oral health has been widely documented [42]. However, in this study, dental attendance was associated with deteriorated OHRQoL.That pattern might reflect perceived treatment need among the study population [43]. This is consistent with results reported previously $[44,45]$. Although pain was the second less commonly reported symptom, it emerged as the strongest predictor of oral impacts among the symptoms investigated both in adjusted and mutually adjusted logistic regression analyses. This is consistent with the multidimensional nature of pain that affect physical, social and psychological well-being $[10,46]$. In the context of oral health, oral pain influences eating, drinking, and other oral every day activities. Conversely, the highly prevalent condition of tooth decay had a small negative impact on OIDP. This might be attributed to the fact that patients learn to cope with commonly occurring symptoms and conditions that become less disabling with recurrence.

The present results corroborate findings with other OHRQoL measures. Generic OHRQoL measures 
Table 6 Models for the association between OML disease groups and OIDP $(n=544)$

\begin{tabular}{|c|c|c|c|c|c|}
\hline $\begin{array}{l}\text { OML disease } \\
\text { groups }\end{array}$ & $\mathrm{N}$ & $\begin{array}{l}\text { OIDP }>0 N=190 \% \\
(n)\end{array}$ & $\begin{array}{l}\text { Unadjusted OR } \\
(95 \% \mathrm{Cl})\end{array}$ & $\begin{array}{l}\text { Adjusted }^{\mathrm{a} O R} \\
(95 \% \mathrm{Cl})\end{array}$ & $\begin{array}{l}\text { Mutually adjusted }{ }^{b} \mathrm{OR} \\
(95 \% \mathrm{Cl})\end{array}$ \\
\hline \multicolumn{6}{|l|}{ Tongue lesions } \\
\hline No & 417 & $34.8(142)$ & 1 & 1 & \\
\hline Yes & 127 & $38.4(48)$ & $1.1(0.7-1.7)$ & $1.0(0.6-1.6)$ & \\
\hline \multicolumn{6}{|l|}{ White lesions } \\
\hline No & 440 & 34.7 (149) & 1 & 1 & \\
\hline Yes & 104 & $39.8(41)$ & $1.2(0.8-1.9)$ & $1.3(0.7-2.1)$ & \\
\hline \multicolumn{6}{|c|}{ Red and blue lesions } \\
\hline No & 484 & $36.1(171)$ & 1 & 1 & \\
\hline Yes & 60 & $32.3(19)$ & $0.8(0.4-1.4)$ & $0.9(0.9-1.0)$ & \\
\hline \multicolumn{6}{|c|}{ Vesiculobullous lesions } \\
\hline No & 513 & $33.5(169)$ & 1 & 1 & 1 \\
\hline Yes & 31 & $72.4(21)^{* *}$ & $5.2(2.2-11.9)$ & $7.4(2.9-18.8)^{* *}$ & $8.2(3.2-20.9)^{* *}$ \\
\hline \multicolumn{6}{|c|}{ Oral ulcerative lesions } \\
\hline No & 520 & $33.9(173)$ & 1 & 1 & 1 \\
\hline Yes & 24 & $77.3(17)^{* *}$ & $6.6(2.4-18.3)$ & $5.7(1.9-16.9)^{*}$ & $6.7(2.2-20.0)^{*}$ \\
\hline \multicolumn{6}{|c|}{ Pigmented lesions } \\
\hline No & 523 & $35.7(183)$ & 1 & 1 & \\
\hline Yes & 21 & $35.0(7)$ & $0.9(0.3-2.4)$ & $0.7(0.2-2.3)$ & \\
\hline
\end{tabular}

*p $<0.05, * * \mathrm{p}<0.001$

a) Adjusted for sex, age, education, perceived health status, dental attendance and medical condition.

b) Adjusted for sex, age, education, perceived health status, dental attendance, medical condition and other OML disease groups.

The sum of the categories listed may not equal the total number due to lack of information.

(OHRQoL-UK measure and OHIP-14 were proven to be valid and reliable in patients with oral lichen planus (OLP) [10]. Moreover, oral health in patients with symptomatic OLP was reported to have an increased burden on their life quality compared to those with nonsymptomatic OLP. Mc Grath et al [8] found that patients with ulcers, erosions and symptomatic oral lesions had bad OHIP-14 scores, suggesting that they had increased quality of life impairments compared to their counterparts with non-symptomatic lesions. Similar results have been presented in studies of patients with Behçet's disease using the OHIP-14 inventory [47]. In another study of UK patients, attending an outpatient oral medicine clinic, Llewellyn et al [9] found that patients with stomatological disease to have higher levels of functional limitations, physical pain and psychological discomfort than the general population. Oral ulceration associated with Behçet's disease and recurrent aphthous stomatitis (RAS) have been reported to impair life satisfaction and the performance of daily activities [11,13]. A Spanish study comparing OHRQoL in patients with OLP with healthy controls concluded that impairments were greatest in the former group of patients across all dimensions of the OHIP inventory [14]. According to this study results, about $30-40 \%$ of the patients with the
OML disease groups of tongue lesions, white lesions, red and blue lesions and pigmented lesions reported oral impacts. On the other hand, the impact frequency among patients suffering oral ulcerative conditions and vesiculobullous diseases amounted to $77 \%$ and $72 \%$, respectively. A previous study revealed that RAS and pemphigus vulgaris were the most frequently occurring diagnosis among oral ulcerative conditions and vesiculobullous diseases in mucocutaneous diseased patients attending the $\mathrm{KTH}$ [5] . Evidence that RAS has the highest impact on patients' quality of life as compared to other oral mucosal diseases in dermatology patients has been shown elsewhere [13]. The present findings suggest that practitioners should notify type and number of OML and reported symptoms when making their treatment plan for this category of patients.

This study suggests that Sudanese patients with mucocutaneous diseases suffer moderate impairments of their OHRQoL, which is measureable by the Arabic version of the generic OIDP inventory. Moreover, eating, emotional problems and cleaning were the most frequently reported impacts, followed by problems with sleeping and speaking across subjects with and without OML. This compares to what has been observed among subjects with other medical conditions as well as with subjects from 
the general adult population in developed and developing countries $[25,40,48]$. The present frequency of OIDP ranging from $30 \%$ to $44 \%$ is comparable to the estimates of a national Greek survey (39\%), but is higher than those reported from national surveys in Norway (18\%) and Great Britain (12\%) $[28,40]$. On the other hand, this figure is lower than those observed in older adults in other cultures (50-60\%) [26], and from dental attendees in Khartoum $(79 \%)$ [31]. The present figures are also lower than those observed among Swedish adult patients (50-54 \%) reporting regular medication according to the Anatomical Therapeutic Chemical classification system and having specific diagnoses of diseases categorized according to the WHO International Classification of Diseases, the ICID-10 [48].

Some limitations should be considered when interpreting the results. First, the cross sectional design restricts ability to make inferences with respect to the direction of the observed associations. Secondly, being a hospital based study; it is not possible to generalize findings to any larger population of mucocutaneous diseased individuals inside or outside Khartoum. Nevertheless, as KTH is the largest public main referral hospital in Sudan, receiving patients referred from all district in Sudan, the dermatology clinic-outpatients may capture the variety in characteristic of patients with skin diseases. In addition, self- reports and a recall period of 6 months can result in underestimation of health consequences, but might provide valid estimate for ultimate impact [49]. Self-selection and non-response bias might have influenced the results as patients were probably more likely to respond when they had OML. The present study suffered from lack of information regarding nonresponders and thus non -response biases are difficult to estimate. Moreover, with respect to the diversity of the types of OML, the present figures might be biased towards those for which people are more inclined to seek treatment, whereas other conditions are less likely to be identified in hospital based prevalence studies. Absence of normative OIDP scores of the general Sudanese adult population, further limits possibility to use the general population as control group. Moreover, it should be acknowledged that the observations related to specific types of OML disease groups were based on small numbers and that the reported impacts cannot be attributed to specific diseases, symptoms and lesions. On the other hand, the generic OIDP scores might be compared across oral diseases and across specific patient groups and the general population. A generic OHRQoL instrument, such as the OIDP could help dermatologists to detect oral impacts, improve the patient doctor communication and provide the basis for better management of the dermatological patients, involving patients' as well as the doctors' perspectives.

\section{Conclusions}

OIDP was more frequently affected among skin diseased patients with than without OML. The frequency of the impacts differed according to the number of type of OML, oral symptoms, and OML disease groups. Dentists and dermatologists should pay special attention to skin diseased patients because they are likely to experience oral impacts on daily performances.

\section{Competing interests}

The authors declare that they have no competing interests.

\section{Acknowledgments}

This work was funded by University of Bergen. The authors wish to thank The Gade Institute, UST and KTH-dermatology department in Sudan for kind cooperation and support of this research, as well as dermatology patients and dentists who helped in data collection and made this work done. Special thanks to dermatologist Adil Basher in designing the skin disease diagnostic sheet and his continued assistance during the data collection. Also we acknowledge the helpful comments of Professor Lisbeth Sviland and senior consultant dermatologist Lisbeth Rustad. We also thank Edith Fick for kind assistance in the laboratory work.

\section{Author details}

${ }^{1}$ Section for pathology, The Gade Institute, University of Bergen, Bergen Norway. ${ }^{2}$ Haukeland University Hospital, Bergen, Norway. ${ }^{3}$ Faculty of Dentistry, University of Science and Technology, Umdurman, Sudan. ${ }^{4}$ Dermatology Clinic, Khartoum Teaching Hospital, Khartoum, Sudan. ${ }^{5}$ Department of Clinical Dentistry, University of Bergen, Bergen, Norway.

\section{Authors' contributions}

NMS was the main author conceived and designed the study, collected data, performed statistical analysis and drafted the manuscript. ACJ was supervising and guiding the whole work and confirmed and approved all the diagnosis of oral lesions. RWA facilitated the field work. HS was the main dermatologist who examined and diagnosed all the patients. ANA was the main-supervisor of the present study, participated and guided the study design and has been actively involved in all stages throughout the work, especially statistical analyses and epidemiological analyses of data. All authors read and approved the final manuscript.

Received: 25 October 2011 Accepted: 8 July 2012

Published: 8 July 2012

\section{References}

1. Budimir J, Mihic LL, Situm M, Bulat V, Persic S, Tomljanovic-Veselski M: Oral lesions in patients with pemphigus vulgaris and bullous pemphigoid. Acta Clin Croat 2008, 47(1):13-18.

2. Hernandez-Perez F, Jaimes-Aveldanez A, Urquizo-Ruvalcaba Mde L, DiazBarcelot M, Irigoyen-Camacho ME, Vega-Memije ME, Mosqueda-Taylor A: Prevalence of oral lesions in patients with psoriasis. Med Oral Patol Oral Cir Bucal 2008, 13(11):E703-708.

3. Schroff J: Primary mouth manifestations of skin diseases. J Dent Res 1930, 10(2):219-231.

4. Bork K: Diseases of the Lips and Mouth. In Braun-Falco's Dermatology. 3rd edition. Edited by Burgdorf WHC, Plewig G, Wolff HH, Landthaler M.: Springer; 2009:1081-1107.

5. Suliman NM, Astrom AN, Ali RW, Salman H, Johannessen AC: Oral mucosal lesions in skin diseased patients attending a dermatologic clinic: a crosssectional study in Sudan. BMC Oral Health 2011, 11(1):24

6. Bowling A: Measuring Health: A Review of Quality of Life Measurement Scales. 3rd edition. Open University Press; 2004.

7. Bernabe E, Marcenes W, Mather J, Phillips C, Fortune F: Impact of Behcet's syndrome on health-related quality of life: influence of the type and number of symptoms. Rheumatology (Oxford) 2010, 49(11):2165-2171.

8. McGrath C, Hegarty AM, Hodgson TA, Porter SR: Patient-centred outcome measures for oral mucosal disease are sensitive to treatment. Int J Oral Maxillofac Surg 2003, 32(3):334-336. 
9. Llewellyn CD, Warnakulasuriya S: The impact of stomatological disease on oral health-related quality of life. Eur J Oral Sci 2003, 111(4):297-304.

10. Hegarty AM, McGrath C, Hodgson TA, Porter SR: Patient-centred outcome measures in oral medicine: are they valid and reliable? Int J Oral Maxillofac Surg 2002, 31(6):670-674.

11. Mumcu G, Ergun T, Inanc N, Fresko I, Atalay T, Hayran O, Direskeneli H: Oral health is impaired in Behcet's disease and is associated with disease severity. Rheumatology (Oxford) 2004, 43(8):1028-1033.

12. Mumcu G, Hayran O, Ozalp DO, Inanc N, Yavuz S, Ergun T, Direskeneli H: The assessment of oral health-related quality of life by factor analysis in patients with Behcet's disease and recurrent aphthous stomatitis. J Oral Pathol Med 2007, 36(3):147-152

13. Tabolli S, Bergamo F, Alessandroni L, Di Pietro C, Sampogna F, Abeni D: Quality of life and psychological problems of patients with oral mucosal disease in dermatological practice. Dermatology 2009, 218(4):314-320.

14. Lopez-Jornet P, Camacho-Alonso F: Quality of life in patients with oral lichen planus. J Eval Clin Pract 2010, 16(1):111-113.

15. Hapa A, Aksoy B, Polat M, Aslan U, Atakan N: Does recurrent aphthous stomatitis affect quality of life? A prospective study with 128 patients evaluating different treatment modalities. J Dermatolog Treat 2011, 22(4):215-220.

16. Vargas-Ferreira F, Piovesan C, Praetzel JR, Mendes FM, Allison PJ, Ardenghi TM: Tooth erosion with low severity does not impact child oral healthrelated quality of life. Caries Res 2010, 44(6):531-539.

17. Tsakos G, Bernabe E, D'Aiuto F, Pikhart H, Tonetti M, Sheiham A, Donos N: Assessing the minimally important difference in the oral impact on daily performances index in patients treated for periodontitis. $J$ Clin Periodontol 2010, 37(10):903-909

18. Rozier RG, Pahel BT: Patient- and population-reported outcomes in public health dentistry: oral health-related quality of life. Dent Clin North Am 2008, 52(2):345-365. vi-vii.

19. Slade GD, Strauss RP, Atchison KA, Kressin NR, Locker D, Reisine ST: Conference summary: assessing oral health outcomes-measuring health status and quality of life. Community Dent Health 1998, 15(1):3-7.

20. Lee GH, McGrath C, Yiu CK, King NM: A comparison of a generic and oral health-specific measure in assessing the impact of early childhood caries on quality of life. Community Dent Oral Epidemiol 2010, 38(4):333-339.

21. Adulyanon S, Vourapukjaru J, Sheiham A: Oral impacts affecting daily performance in a low dental disease Thai population. Community Dent Oral Epidemiol 1996, 24(6):385-389.

22. Adulyanon S, Sheiham A: Oral Impact on Daily Performances. In Measuring Oral Health and Quality of Life. Edited by Slade GD. North Carolina: Department of Dental Ecology, School of Dentistry, University of North Carolina; 1997:152-160

23. Badley EM: he ICIDH: format, application in different settings, and distinction between disability and handicap. A critique of papers on the application of the International Classification of Impairments, Disabilities, and Handicaps. Int Disabil Stud 1987, 9(3):122-125.

24. Locker D: Measuring oral health: a conceptual framework. Community Dent Health 1988, 5(1):3-18.

25. Kida IA, Astrom AN, Strand GV, Masalu JR, Tsakos G: Psychometric properties and the prevalence, intensity and causes of oral impacts on daily performance (OIDP) in a population of older Tanzanians. Health Qual Life Outcomes 2006, 4:56.

26. Srisilapanan $P$, Sheiham A: The prevalence of dental impacts on daily performances in older people in Northern Thailand. Gerodontology 2001 18(2):102-108

27. Sheiham A, Steele JG, Marcenes W, Tsakos G, Finch S, Walls AW: Prevalence of impacts of dental and oral disorders and their effects on eating among older people; a national survey in Great Britain. Community Dent Oral Epidemiol 2001, 29(3):195-203.

28. Tsakos G, Marcenes W, Sheiham A: Cross-cultural differences in oral impacts on daily performance between Greek and British older adults. Community Dent Health 2001, 18(4):209-213.

29. de Oliveira CM, Sheiham A, Tsakos G, O'Brien KD: Oral health-related quality of life and the IOTN index as predictors of children's perceived needs and acceptance for orthodontic treatment. Br Dent J 2008, 204 (7):1-5. discussion 384-385.

30. Nurelhuda NM, Ahmed MF, Trovik TA, Astrom AN: Evaluation of oral health-related quality of life among Sudanese schoolchildren using Child-OIDP inventory. Health Qual Life Outcomes 2010, 8:152.
31. Ali WM: Assessment of oral impact on daily performance, dental caries, oral hygiene and periodontal disease among a group of adult dental patients Master thesis of Philosophy in Oral sciences.: University of Bergen; 2011.

32. Kramer IR, Pindborg JJ, Bezroukov V, Infirri JS: Guide to epidemiology and diagnosis of oral mucosal diseases and conditions. World Health Organization. Community Dent Oral Epidemiol 1980, 8(1):1-26.

33. Organization WH: Oral health Surveys: Basic Methods. 4th edition. Geneva: Organization WH; 1997.

34. Axell T, Pindborg JJ, Smith CJ, van der Waal I: Oral white lesions with special reference to precancerous and tobacco- related lesions: conclusions of an international symposium held in Uppsala, Sweden, May 18-21 1994. International Collaborative Group on Oral White Lesions. J Oral Pathol Med 1996, 25(2):49-54

35. Axell T: A prevalence study of oral mucosal lesions in an adult Swedish population. Odontol Revy Supp/ 1976, 36:1-103.

36. Streiner David L, Norman GR: Health Measurement Scales. A practical guide to their development and use 2nd edn. 2nd edition. New York: Oxford University Press Inc; 1995

37. Al-Jundi MA, Szentpetery A, John MT: An Arabic version of the Oral Health Impact Profile: translation and psychometric properties. Int Dent J 2007, 57(2):84-92

38. McGrath C, Alkhatib MN, Al-Munif M, Bedi R, Zaki AS: Translation and validation of an Arabic version of the UK oral health related quality of life measure (OHQoL-UK) in Syria, Egypt and Saudi Arabia. Community Dent Health 2003, 20(4):241-245.

39. Daradkeh S, Khader YS: Translation and validation of the Arabic version of the Geriatric Oral Health Assessment Index (GOHAI). J Oral Sci 2008, 50 (4):453-459.

40. Astrom AN, Haugejorden O, Skaret E, Trovik TA, Klock KS: Oral Impacts on Daily Performance in Norwegian adults: the influence of age, number of missing teeth, and socio-demographic factors. Eur J Oral Sci 2006, 114 (2):115-121.

41. Riordain RN, McCreary C: Validity and reliability of a newly developed quality of life questionnaire for patients with chronic oral mucosal diseases. J Oral Pathol Med 2011, 40(8):604-609.

42. Locker D: Does dental care improve the oral health of older adults? Community Dent Health 2001, 18(1):7-15.

43. Tsakos G, Marcenes W, Sheiham A: Evaluation of a modified version of the index of Oral Impacts On Daily Performances (OIDP) in elderly populations in two European countries. Gerodontology 2001, 18(2):121-130.

44. Mtaya M, Astrom AN, Tsakos G: Applicability of an abbreviated version of the Child-OIDP inventory among primary schoolchildren in Tanzania. Health Qual Life Outcomes 2007, 5:40.

45. Masalu JR, Astrom AN: Social and behavioral correlates of oral quality of life studied among university students in Tanzania. Acta Odontol Scand 2002, 60(6):353-359.

46. Becker N, Bondegaard Thomsen A, Olsen AK, Sjogren P, Bech P, Eriksen J: Pain epidemiology and health related quality of life in chronic nonmalignant pain patients referred to a Danish multidisciplinary pain center. Pain 1997, 73(3):393-400.

47. Mumcu G, Niazi S, Stewart J, Hagi-Pavli E, Gokani B, Seoudi N, Ergun T, Yavuz S, Stanford M, Fortune F, et al: Oral health and related quality of life status in patients from UK and Turkey: a comparative study in Behcet's disease. J Oral Pathol Med 2009, 38(5):406-409.

48. Ostberg AL, Andersson P, Hakeberg M: Oral impacts on daily performances: associations with self-reported general health and medication. Acta Odontol Scand 2009, :1-7.

49. Mock C, Acheampong F, Adjei S, Koepsell T: The effect of recall on estimation of incidence rates for injury in Ghana. Int J Epidemio/ 1999, 28 (4):750-755.

\section{doi:10.1186/1472-6831-12-19}

Cite this article as: Suliman et al.: Influence of oral mucosal lesions and oral symptoms on oral health related quality of life in dermatological patients: a cross sectional study in Sudan. BMC Oral Health 2012 12:19. 